\title{
Progress in the Development of Fast Timing Micro-Pattern Gaseous Detector
}

\author{
Christos Roskas ${ }^{* 1}$, Rui de Oliveira ${ }^{2}$, llaria Vai $^{3}$, Marcello Maggi ${ }^{4}$, Michael Tytgat ${ }^{1}$, \\ Piet Verwilligen ${ }^{4}$ \\ ${ }^{1}$ Ghent University, ${ }^{2}$ CERN,${ }^{3}$ Universitá di Bergamo \& INFN Pavia, ${ }^{4}$ INFN Bari \\ E-mail: christos.roskasecern.ch, Rui.De.oliveira@cern.ch, \\ Ilaria.Vai@cern.ch, Marcello.Maggi@cern.ch, Michael. Tytgat@cern.ch, \\ Piet.Verwilligen@cern.ch
}

\begin{abstract}
Advances in the photo-lithographic techniques during the last twenty years have led to the development of micro-pattern gaseous detectors (MPGD). Their main features include high rate capability and radiation hardness, excellent spatial resolution, good time resolution, reduced radiation length and possible flexible geometries. In recent years the further development of MPGDs concentrated on using resistive materials to build compact spark-protected detectors. The use of resistive materials also opened the possibility to make electrically transparent structures with external signal pick-up electrodes. This allowed for a new idea to improve the time resolution through a multi-layered detector, consisting of alternating drift and amplification regions, where the fastest signal determines the detection time. This so-called Fast Timing MPGD (FTM) was firstly introduced by Rui de Oliveira et al. in 2015 [1] and aims to combine both the high spatial resolution and the high rate capability of a MPGD with a high time resolution of the order of 300 ps. Here, we introduce the design of a new single-layer prototype to test the gain of the amplification structure. Preliminary results on the detector characterization will be shown.
\end{abstract}

European Physical Society Conference on High Energy Physics - EPS-HEP2019 -

10-17 July, 2019

Ghent, Belgium

${ }^{*}$ Speaker. 


\section{Introduction}

The Fast Timing Micro-Pattern Gaseous Detector [1, 2] (Fast Timing MPGD - FTM) was conceived to improve the time resolution of traditional MPGD detectors of at least an order of magnitude, keeping other typical MPGD characteristics as excellent spatial resolution, high rate capability, excellent radiation hardness and flexible geometries. The idea of the FTM is to improve the time resolution in the sub-ns scale by segmenting the drift gap in multiple sub-mm drift gaps, each coupled to its own amplification stage. In a standard MPGD, just like GEMs, the time resolution is determined by fluctuations of the position of primary ionization with respect to the amplification structure and improves with the drift velocity. Therefore smaller drift region leads to a smaller uncertainty on the position of the first ionization in the drift gap due to the reduction of the gap itself, which leads to a total reduction on time resolution proportional to the number of amplification stages $N_{D}$ employed [1]:

$$
\sigma_{t}=\left(\lambda u_{d} N_{D}\right)^{-1}
$$

\section{Structure of the FTM prototype}

This FTM prototype has been designed with the aim of fully characterizing a single layer before proceeding to multi-layer amplification stages. A single layer is made of 3 kapton foils of $50 \mu \mathrm{m}$ thickness: a copper cladded foil, the FTM amplification foil and a non-etched DLC coated polyimide. The layout of the prototype is shown in Fig.1. The copper cladded foil is used as the drift electrode and it is placed 5mm away from the DLC amplification foil to create the drift gap. The amplification foil has a WELL structure in which the small diameter (top side) is in the range of $35-40 \mu \mathrm{m}$ and the big diameter (bottom side) is $85-90 \mu \mathrm{m}$. In this prototype the active (holepatterned) area is circular with a diameter of $30 \mathrm{~mm}$. Below the DLC perforated foil lies a DLC coated foil which works as the anode to set a ground potential. From that foil we are able to read the current that is produced inside the amplifiaction region. The readout board (RO) consists of 200 strips of $400 \mu \mathrm{m}$ width spaced $500 \mu \mathrm{m}$ apart, read by two panasonic-to-LEMO connectors.

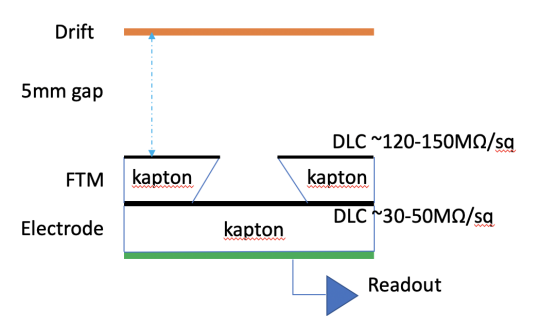

Figure 1: Schematic overview of a single layer of the FTM prototype: on top the drift electrode, followed by a $5 \mathrm{~mm}$ drift gas gap, then the well-like amplification structure made of a perforated kapton foil with DLC coating on top of the third DLC coated kapton foil that provides ground potential, mounted on top of a readout board with $400 \mu \mathrm{m}$ strips at $500 \mu \mathrm{m}$ pitch.

The values of the impedance on the resistive layers have been measured for several locations on the surface of the foil using an insulator tester (MEGGER MIT 420 [8]) and are $30-50 \mathrm{M} \Omega / \square$ for the bottom DLC electrode and $120-150 \mathrm{M} \Omega / \square$ for the top DLC electrode. 


\section{Foil production}

The production of the amplification foil of the FTM follows the single-mask procedure for the production of GEM foils $[10,11]$. The base flexibile-cupper-clad laminate (FCCL) is a $50 \mu \mathrm{m}$ polyimide foil, covered on one side with $5 \mu \mathrm{m} \mathrm{Cu}$ and on the other side with $100 \mathrm{~nm}$ of DLC. A thin $\mathrm{Cr}$ layer improves the adhesion of the $\mathrm{Cu}$ layer on the Polyimide foil. The DLC was deposited through the magnetron sputtering technique [9]. To protect the DLC during the etching procedure it was covered first with a thin $\mathrm{Cr}$ layer, thereafter with a $10 \mu \mathrm{m}$ thick $\mathrm{Cu}$ layer. Figure 2 (left) shows the foil before the start of the etching while figure 2 (right) shows a fully etched FTM amplification foil.

The photolitographic methods used for the perforation of polyimide foils require a thin $\mathrm{Cu}$ layer. Therefore the etching procedure necessarily starts from the bottom side of the FCCL where only two materials needed to be manipulated. The goal of the etching is to have holes of $90-$ $100 \mu \mathrm{m}$ on the bottom side and around $40-50 \mu \mathrm{m}$ on the top (DLC) side.

A first step before starting the etching procedure is a thorough cleaning of the foil to remove unwanted remnants that might exist due to the several cladding depositions. This step is performed in a sandblasting machine which uses a mixture of water with fine stone grains. Subsequently the foil is dried in an oven at $60^{\circ} \mathrm{C}$ for about 15 minutes.

The second step is the lamination process, in which a photo-resistive layer is deposited on the both sides of the foil. The laminated foil is then inserted in the photo-lithographic machine where the desired mask is printed. Afterwards the foil is developed with UV light in order to be pre-etched. At the end of this step the foil is placed again at the oven at $80^{\circ} \mathrm{C}$ for about 10 minutes.

When the foil is completely dried, the photo-resistive layer is removed and the foil itself is placed in the machine that develops resist to the areas which need to be protected from the $\mathrm{Cu}$ etching. The foil is placed it is thoroughly cleaned with water and placed again to the oven in order to dry-out.

Now the hole pattern has been transferred to the $\mathrm{Cu}$ layer of the FCCL and the foil is put in the $\mathrm{Cu}$ etching machine. The $\mathrm{Cu}$ area not covered by the photo-resist is removed with $\mathrm{FeCl}_{3}$ in a two-step redox reaction. After several iterations the $\mathrm{Cu}$ is removed from the areas where the holes were patterned. After the removal of the cupper, the thin Cr layer is removed in a special machine with warmed permanganate solution. The diameter of the hole formed during the etching process depends on the initial concentration of the chemical solution of ethylene diamine and potassium hydroxide $(\mathrm{KOH})$ and the time the foil is submerged. Since the concentration of the solution is subject to variations, prior to the actual etching a test etching of a small size sample is performed to estimate the required duration. For our deposition this time was determined to be 8 minutes.

Once finished the etching initiated from the bottom, one completes the etching starting from the top (DLC side) of the polyimide. A major difference however is due to the removal of the Cr since the permanganate solution [12] acts also on the DLC removing it to large extend. For this reason a softer dissolver was used. It consist of three components: amonium cerium(IV) nitrate $150 \mathrm{gr}$, dissolved in $1.125 \mathrm{~L}$ of water and adding $35 \mathrm{~mL}$ of acetic acid. The results on the removal of Cr without affecting the DLC were satisfying and eventually we succeed in creating a operational foil ready to be tested in a prototype. The surface resistivity of the foil was measured to be $120-150 M \Omega / \square$. Fig. 2 (right) shows the finalized FTM foil mounted on the FTM prototype. 


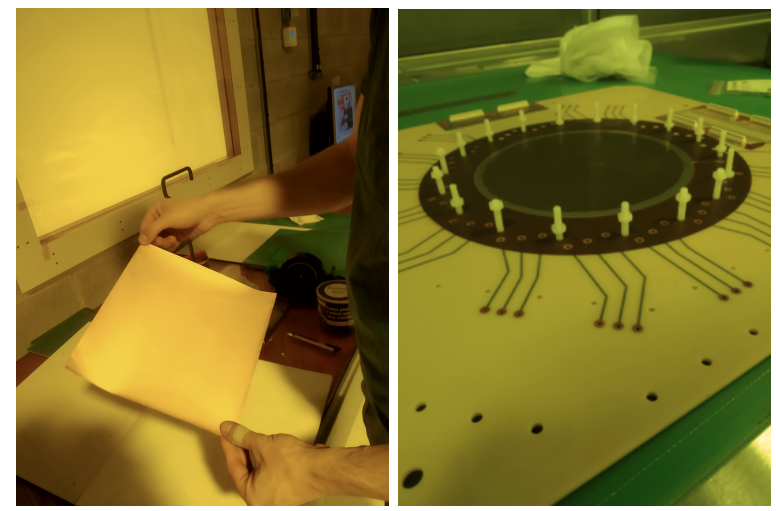

Figure 2: The FTM foil after the whole process of etching, placed on the RO board during the assembly of the prototype.

\section{Measurements - Results}

The prototype was tested at Ghent in the GEMLab. The main goal of those tests was to characterize a single layer. A full characterization of a single requires to perform the following tests: signal waveform observation with $X$-ray, linearity of count rate with X-ray intensity, measurement of the effective gain

The prototype was initially powered on with the HV module CAEN 1470A [3], a 2-channel power supply which was remotely controlled via a labview software for real-time monitoring and data acquisition. Then the power supply was changed with the CAEN 1471H [4] which has a highaccuracy mode providing current resolution of $50 \mathrm{pA}$. For the irradiation of the prototype a Silver (Ag) target X-ray gun was used (AMPTEK mini-X) [5]. The current that is collected from the anode (DLC electrode) was read out by the pico-ammeter, Keithley 6487 [6]. To read 200 strips on the oscilloscope Panasonic-to-LEMO connectors were used to unify the signal and send to the ORTEC 142PC pre-amplifier [7].

Fig. 3 shows the dependence of the current that is collected on the anode, the DLC coated electrode below the amplification foil, in two cases. In the first one the drift field is kept stable at $0.2 \mathrm{kV} / \mathrm{cm}$ so as the amplification field at $90 \mathrm{kV} / \mathrm{cm}$ while the X-ray intensity was increasing. In the second the amplification field is constant at $90 \mathrm{kV} / \mathrm{cm}$ as well as the X-ray intensity while this time the drift field value is increasing. The results show that indeed there is a linear relation between the collected current and the X-ray intensity and also that the optimal drift region for maximum collection efficiency is at $2 \mathrm{kV} / \mathrm{cm}$.

The effective gain is estimated with the formula: $G\left(V_{F T M}\right)=\frac{I_{\text {anode }, \text { amp }}\left(V_{F T M}\right)}{I_{\text {anode, }, \text { inization }}} \cdot \frac{I_{\text {xray,ionization }}}{I_{\text {xray }}\left(V_{F T M}\right)}$

where $I_{\text {anode,amp }}$ is the average current induced on the anode and is dependent on the amplification field, $I_{x r a y}\left(V_{F T M}\right)$ is the value of the x-ray gun current set for the measurement, $I_{\text {anode, ionization }}$ is the ionization current. In order to calculate the ionization current we perform measurements in which there is a steady X-ray flux while the amplification voltage is changing. Primary results are given in Fig. 4 below:

In Fig. 4(left) the current which is plotted is the result of the subtraction between the current collected on the anode while irradiated and while not irradiated: $\left\langle I_{\text {tot }}\right\rangle=\mid\left\langle I_{\text {anode }}\right\rangle_{\text {sourceON }}-<$ 

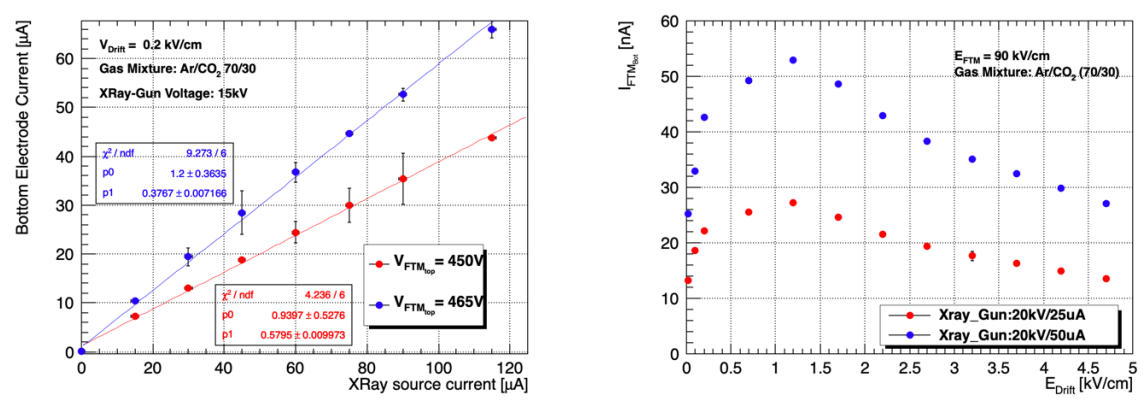

Figure 3: (Left) Collected current on the anode after the amplification for constant drift field and 2 setpoints of amplification field. (Right) Collected current on the anode for increasing drift field while keeping the amplification field constant and the X-ray beam intensity steady.
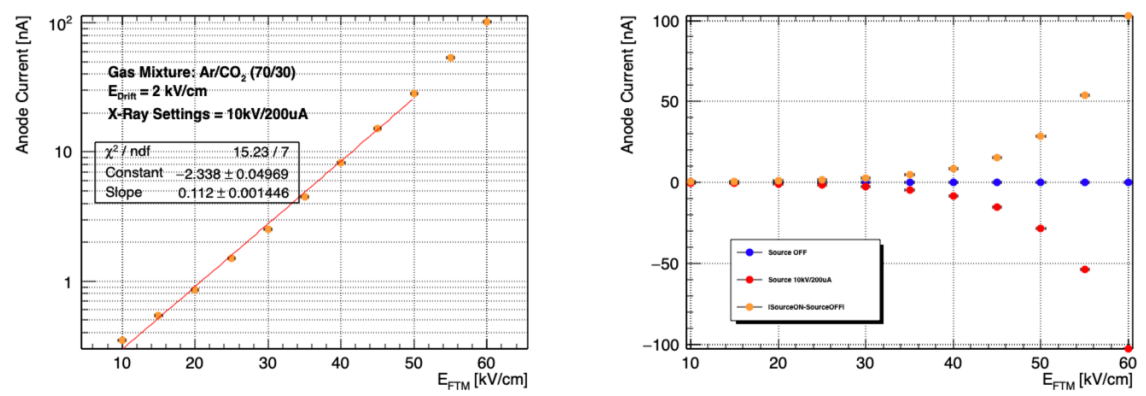

Figure 4: (Left) Current drawn by the DLC bottom electrode while keeping a steady flux and increasing the voltage on the FTM foil. (Right) Current drawn on the anode while: not irradiated (blue dots), irradiated(red dots), absolute value of their subtraction (orange dots).

$I_{\text {anode }}>_{\text {sourceOFF }} \mid$

A plateau is expected in the region up to $15 \mathrm{kV} / \mathrm{cm}$ right before the amplification in the hole sets in. However in Fig. 4 we see an exponential increase on the current starting from $10 \mathrm{kV} / \mathrm{cm}$. For this reason, before proceeding to a effective gain estimation, we are conducting more precise measurements as it is crucial to have the best possible precision on the ionization current measurement.

\section{Conclusions}

A procedure to etch DLC-covered polyimide foil was established, leading to a high quality perforated foil with constant hole diameter. Several tests were performed focusing mostly on the verification of linearity of the counting rate with respect to the X-ray intensity, the effective gain measurement and also the observation of the signal using X-ray source. The results showed that the prototype indeed has a linear behaviour with the X-ray intensity however the gain measurements are still ongoing and we have not been able to observe a signal induced in the readout strips. Therefore future research will be focused on reducing the material between the bottom of the readout hole and the strips to improve the signal pick-up. Furthermore once we will have a precise gain estimation we would like to operate the foils at electric field that will reach an amplification of $10^{4}$. 


\section{References}

[1] R. De Oliveira, M. Maggi and A. Sharma, "A novel fast timing micropattern gaseous detector: FTM," arXiv:1503.05330 [physics.ins-det].

[2] D. Abbaneo et al. , "R\&D on a new type of micropattern gaseous detector: The Fast Timing Micropattern detector ", Nucl. Instrum. Meth. A 845 (2017) 313. doi:10.10.16/j.nima.2016.05.067

[3] N1470 4Ch Reversible 8kV/3mA (8W) NIM HV Power Supply Module (USB).

https://www. caen.it/products/n1470/

[4] N1471H 4Ch Reversible 5.5kV/20 $\mu$ A NIM HV Power Supply High Accuracy Module (USB). https://www.caen.it/products/n1471h/

[5] Amptek Mini-X X-Ray Tube System for XRF.

https://www. amptek.com/products/x-ray-sources/mini-x2-ray-tube

[6] Model 6487 Picoammeter/Voltage Source Manual Reference Manual Manual

https://www.tek.com/low-level-sensitive-and-specialty-instruments / series-6400-picoammeters-manual/model-6487-picoammeter

[7] ORTEC 142PC Preamplifier

https:

//www.ortec-online.com/products/electronics/preamplifiers/142pc

[8] Megger MIT420

https://megger.com//cat-iv-insulation-testers-mit400/2-series

[9] You Lv, Yi Zhou, Jianbei Liu, Ming Shao, Zhiyong Zhang, Guofeng Song, Xu Wang, "Production and performance study of Diamond-Like Carbon resistive electrode in MPGD,"

Nuclear Instruments and Methods in Physics Research Section A: Accelerators, Spectrometers, Detectors and Associated Equipment, 2019, 162759,

ISSN 0168-9002,

https://doi.org/10.1016/j.nima.2019.162759.

(http://www.sciencedirect.com/science/article/pii/s0168900219312148)

[10] S. D. Pinto et al., A large area GEM detector, 2008 IEEE Nuclear Science Symposium Conference Record, Dresden, Germany, 2008, pp. 1426-1432.

[11] S. Duarte Pinto and R. De Oliveira. A method of manufacturing a gas electron multiplier. Patent Wipo WO/2009127220

[12] Permanganate https://en.wikipedia.org/wiki/Permanganate 